\title{
Bandwidth Estimation Tools and Techniques: A Review
}

\author{
Mukta Airon ${ }^{\text {[orcid.org/0000-0002-0637-0382] }}$ and Neeraj Gupta ${ }^{\text {[orcid.org/0000-0002-9650-4988] }}$ \\ K. R. Mangalam University, Gurgaon, India \\ mukta.mittal2006@gmail.com, neerajgupta3729@gmail.com
}

\begin{abstract}
Wireless communication has proliferated due to the colossal augmentation of smart phones, mobile phones, tablets and laptops etc. Multimedia applications such as live videos, audios, still images, animated graphics and live communications all requires accompaniments of quality of service (QoS) as well as reliable end-to-end transfer of data. Information about the availability of resources in channel is must in order to improve the QoS. QoS can be determined in terms of capacity of channel, available bandwidth (ABW), and bulk transfer capacity (BTC). Bandwidth availability is a key check for improving the QoS in a network. The performance of a multimedia application is directly affected by the bandwidth availability. One of the most important QoS characteristic is ABW at a wireless route and it can be demarcated as least unused capacity of links instituting a network route. Since now there have been many bandwidth estimation techniques are available in the literature to increase the network performance. Bandwidth estimation techniques have been arranged into three leading classifications: i) Active probing bandwidth estimation techniques, ii) Passive bandwidth estimation technique and iii) Model based bandwidth estimation techniques. Each of these techniques are briefly discussed in this paper.
\end{abstract}

Keywords: available bandwidth estimation techniques; active bandwidth estimation techniques; passive bandwidth estimation techniques; model based bandwidth estimation techniques 


\section{INTRODUCTION}

Wireless Ad hoc networks are temporary, decentralize, infrastructure less, quick and easy way of networking used in the situation where it is difficult to set up networks through cabling. Now a day, several applications generate multimedia data like live videos, audios, animated graphics and live communications etc., all these applications necessitate the backing of guaranteed services by the network. Providing QoS in wireless ad hoc networks has attained much attention in recent years. QoS assimilate through some efforts like controlling admissions aimed to render guarantee to the applications in terms of delay, jitter, bandwidth or packet loss etc. Other solution is QoS routing, i.e. electing the best path out of all the possible routes. In either case, for ensuring QoS a precise assessment of obtainable resources is mandatory. Therefore, it is clamorous task to obtain the accurate information of ABW in wireless ad hoc networks. The IEEE standard defined for wireless LANs (WLAN) is 802.11. This standard postulates two practices of accessing the medium, Distributed coordination function (DCF) and Point coordination function (PCF). The mandatory DCF based on carrier sense multiple access with collision avoidance mechanism (CSMA-CA) which is a MAC protocol is used in ad hoc networks. Number of works had been done in the area of ABW estimation in wireless ad hoc networks in the previous literatures but none of them has been standardized till now. The term ABW is demarcated as the vacant bandwidth over the total amount of time referred to as the estimation period. The bandwidth available to a route is defined as the minimum of the ABW's of various links constituting the route. Bandwidth estimation techniques have been arranged into three leading classifications: Active or probe based bandwidth estimation technique, Passive or sensing based bandwidth estimation technique, analytical or model based bandwidth estimation technique.

\subsection{Need for ABW Estimation}

In wireless networks, valuation of $\mathrm{ABW}$ is an ambitious task. These networks are different in characteristics as compared with the wired networks. Factors those effects on the $\mathrm{ABW}$ in wireless networks are dynamic links, shared medium among neighbouring nodes, channel fading and interference from the physical hindrances. These elements do not exist in wired networks, so it's enigmatic task to evaluate the bandwidth available in wireless networks. The indices pertaining to bandwidth are: capacity of link/route, ABW and bulk transfer capacity (BTC). The Link or route capacity of the channel can be demarcated as the maximum amount of data that can be sent over a link or route from source to the destination, whereas the ABW is the extent of residual bandwidth after the cross traffic in the medium. BTC can be stated as highest bandwidth conquered by a single TCP connection.

The capacity of links in wireless networks depends on the size of packet as well as the amount of cross-traffic present in the network. The whole capacity could not be consumed in the transmission of data packets as a portion of this capacity is paid out in the overheads like initiating communications, control message overheads, inter-frame spacing and interference generated by neighbors. Estimation of ABW improves the network's performance, regulate the admission of new flows and improve he QoS provided by the networks.

\subsection{Organization of the paper}

This paper describes the succinct overview of tools utilized for estimating the ABW and techniques presented by the previous literatures. Further content in this paper is systematized as follows: Section 2 provides an insight of Active/ probe-based bandwidth estimation techniques. Following this section 3 describes the passive / sensing -based bandwidth estimation techniques. Analytical / model-based bandwidth estimation techniques are described in the section 4. 


\section{Active/Probe-based Bandwidth Estimation Tools and Techniques}

Active techniques for estimation of ABW are also called Probe-based bandwidth estimation techniques. Here, the sender sends mock-up packets called as probe packets to the receiver so as to create probing traffic on the network thereby knowing the network characteristics. These techniques can be further categorized into four sub-categories as briefly stated below:

\subsection{Active bandwidth estimation techniques}

- Variable Packet Size (VPS) Probing technique [18]

This technique is used to assess the capacity of each hop within a route. Notion behind this approach is to measure the round trip time (RTT) from the source to each hop of the route as a function of probing packet size. VPS utilizes the IP header's "Time to Live" (TTL) field for compelling the expiration of probing packets at a targeted hop and each hop sends an error message corresponding to internet control message protocol (ICMP) time exceeded to the source node. RTT to that targeted hop is calculated by the source based on the ICMP error message. Each hop's capacity is calculated with respect to probe packet size. The result affirms that there is a linear increase in RTT w.r.t the probe packet size as well as number of hops.

- $\quad$ Packet Pair / Train Dispersion (PPTD) [19, 20]

This approach is also called Probe Gap Model (PGM) or direct probing technique. In this technique, a mathematical equation is derived between the ABW and the sending and receiving gaps between the probing packets, thereby by simply measuring these gaps the $\mathrm{ABW}$ can be calculated. If the probe packet sending rate is bottleneck link capacity $\mathrm{C}$, then $\mathrm{ABW}$ is obtained as:

$$
A B=C(2-C / R)
$$

Where, $\mathrm{R}$ is the dispersion rate. Cross traffic may get in between the probe packets, therefore in order to minimize cross-traffic within the network two probe packets are sent back to back to evaluate the ABW. The second probe packet is delayed w.r.t the first one due to bottleneck link capacity. PGM's accuracy is based on the amount of cross-traffic on the path. PGM is not useful in the multi-hop because of its limitation to single bottleneck link.

- $\quad$ Self-Loading Periodic Streams (SLoPS) [21, 22]

This technique calculates end-to-end ABW by monitoring probe packet's one way delay where probe packets of equal size (a "periodic stream ") are sent to path under consideration at a steady rate. The probe packet send rate is compared w.r.t. the ABW. The successive probe packet's one way delay increases if the rate of probe packets greater than the path's ABW. Otherwise, one way delays will not increase. Sender tries to match the sending rate of probe packets with the ABW. The sender adjusts the rate of successive probe packet trains and probes the path, while each periodic stream's one way delay information is stored by the receiver. Further network load is adjusted by the sender in order to ensure only single periodic stream is carried by the network path. Sender adjusts the traffic rate of probing to lesser than $1 / 10^{\text {th }}$ of the path's ABW. SLoPS measures the variation of the ABW of the different periodic streams and notices the stream's one way delay. A fair increasing/decreasing trend is not shown by SLoPS. The grey region reported by SLoPS is corresponding to the deviations in ABW during measurement. SLoPS have fewer overheads as compared to other techniques and it is also fast and accurate ABW estimation technique.

- $\quad$ Trains of Packet Pairs (TOPP) [24]

This uses dispersion approach to calculate the network path's ABW by gradually increasing rate of probe packets. TOPP repeatedly sends the train of packet pair at gradual rate between source and sink node. Each pair's input gap is variated thereby creating a variation in Rate. $\mathrm{ABW}$ is calculated as the maximum rate up to which the input sent from source is smaller than the measured rate at the destination. The offered rate of the packet pair is:

$$
\mathrm{R}_{\mathrm{o}}=\mathrm{L} / \Delta_{\mathrm{s}}
$$


Where, $\Delta_{\mathrm{s}}$ is the initial dispersion of packet pair sent from source to sink; and $\mathrm{L}$ is the probe packet size in bytes. The incoming packet will be queued up after the first probing packet pair and the receiver will measure the rate $R_{m}<R_{o}$, if $R_{o}$ is more than the end-to-end ABW. Otherwise, TOPP considers that receiver will receive the packet pair at the same rate as it had at the sender i.e. $R_{m}=R_{o}$. Notion behind TOPP is basically similar to SLoPS. The way measurements are statistically processed creates differences between these two techniques. The offered rate grows linearly in TOPP, whereas in order to adjust it binary search is used in SLoPS. An additional feature in TOPP is that, path's tight link capacity can also be estimated. The actual capacity of the path may be lesser than this measured capacity if the narrow link and tight link are not the same. During measurement the deviations in ABW can be traced by TOPP and SLoPS. The network path is overloaded in SLoPS and TOPP by self-induced congestion. In both techniques, all the routers in the path are assumed to follow Firstin-first-out (FIFO) sequencing and the average rate of cross-traffic variation is sluggish and is steady during measurement period. The simulation results affirm the handling of single and multiple hops with varying bandwidth and cross-traffic by TOPP. ABW of any path in communication network is estimated by TOPP, particularly in wired networks.

\subsection{Active bandwidth estimation tools}

Depending on the type of methodology adopted for estimation of bandwidth available like per-hop Capacity Estimation Tools, End-to-End Capacity Estimation Tools, End-To End ABW Estimation Tools and Bulk Transfer Capacity Estimation Tools, various tools for active bandwidth estimation could be employed. Per-hop capacity estimation tools are used for estimating each hop's capacity within the path in VPS probing technique. End-To-End capacity estimation tools are employed for evaluating the narrow link's capacity within an end-to-end network path under the packet pair methodology. Under self-loading periodic streams approach, End-to-End ABW estimation tools are used. Tools uses for bulk-transfer capacity estimation are Treno and cap. The active bandwidth estimation tools are classified as shown in Fig1.

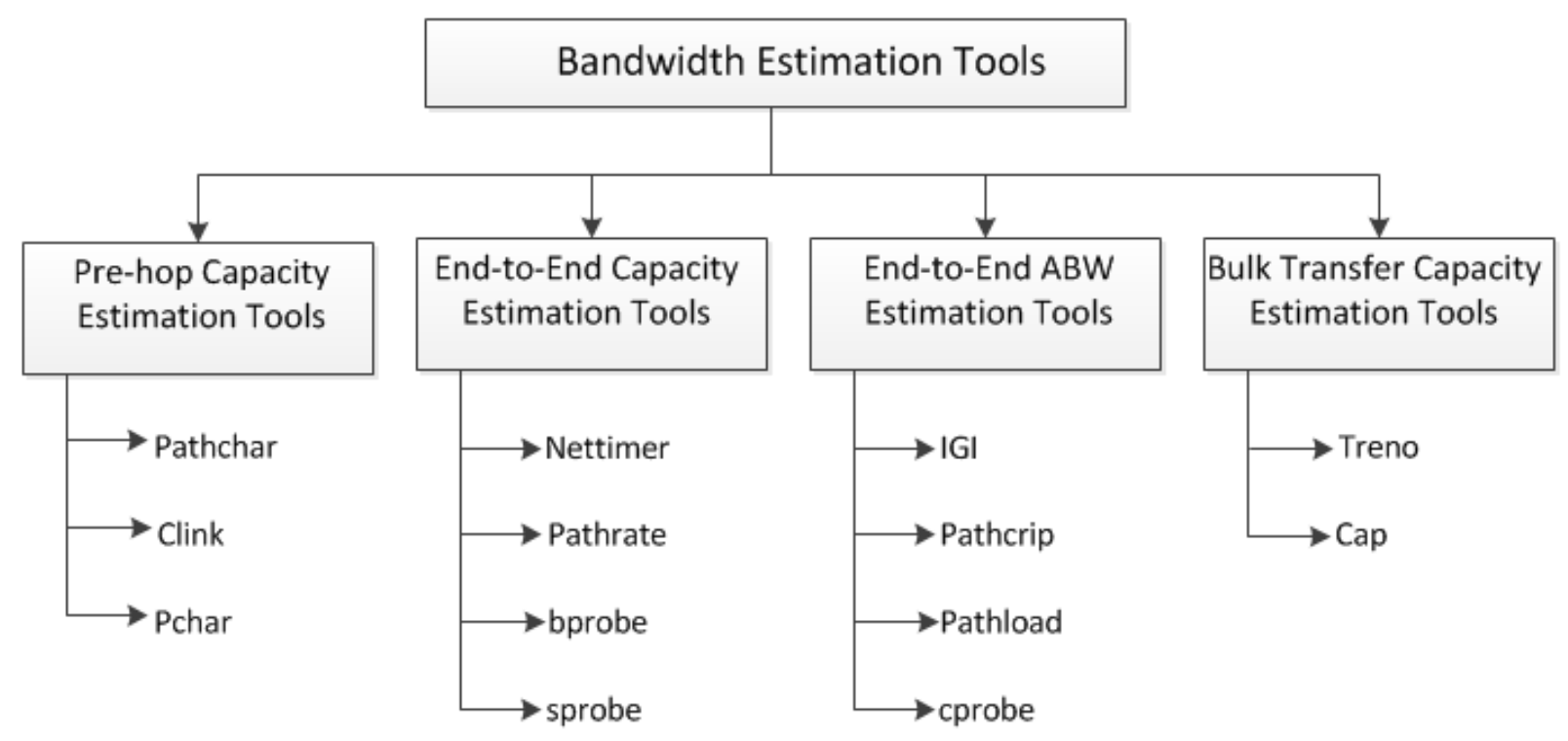

Fig1. Classification of Bandwidth Estimation Tools 


\subsubsection{Per-hop capacity Estimation Tools}

- $\quad$ Pathchar [23]

This tool is used for determining the properties of an Internet Link. Within an internet path, the link with least ABW is considered as the representative for the whole path's ABW. For this estimation, source host sends packets and their round trip time (RTT) is measured. Original sender node fills the TTL (Time-To- Live) entry in the packet that is decremented by one at each intermediate router. If the TTL entry reaches zero, this packet is discarded and correspondingly an error message (ICMP TTL expired) is returned to sender. It is possible to estimate the per hop bandwidth, per hop delay, queue time and collision rate.

- $\quad$ Clink [25]

VPS probing technique use Clink as an open source tool supporting only Linux platform. Unlike Pathchar, Clink uses an "even-odd" technique to provide interval capacity estimates. On experiencing routing instability, this tool starts gathering information from all paths it encounter till at least one of the path provides sufficient information to conclude a statistically noteworthy estimate.

Here, a single source sends UDP packets one by one at an interval of 10 times the RTT of previously sent packet in order to measure the latency and bandwidth of internet links. These probing packets creates overhead on the network and at the recipient machine which under worst case scenario is $1 / 11^{\text {th }}$ of the network's capacity.

- Pchar [26]

Pchar using three different regression algorithms for the RTT measurements against the probe packet size. This tool is portable to UNIX platform. Pchar using VPS probing technique. To obtain the kernel level timestamp Libcap is used.

\subsubsection{End-to-End Capacity Estimation Tools}

- $\quad$ Nettimer [27]

It passively monitors the bandwidth of poorest link within a path in real time fashion. For quantitatively measuring the results of investigations "libdpcap distributed capture library" is employed. This tool can calculate bandwidth in both directions by increasing the number of packet capture hosts from one to two. The employed library allows Nettimer program to competently capture packets at remote hosts undergoing local enormous measurement calculations. Nettimer is based on the notion to run tcpdump traces on the machines pair during transfer between them and alternating the bottleneck link bandwidth, path length and workload thereby analyzing the results. This methodology comprises of the topology and environment within network, the hardware and software, precision of assessment, workload induced due to network application. Results depicts an error lesser than $10 \%$ in most of the scenarios however reaching maximum $41 \%$ error in worst case.

- $\quad$ Pathrate [28]

This is a capacity estimation tool. Pathrate deals with two end point methodology, so it is more accurate than other estimation tools. In this source and the sink both cooperate with each other for measurement of ABW. Pathrate deploys TCP connection known as control channel for exchanging control info in-between end points. This tool sends probing packets using UDP. Measuring process ignores any loss of packet pair or trains thereby avoiding path congestion. User-level time stamping is used at the reception part of Pathrate. The estimated bandwidth is greater than the network interface bandwidth at receiving host. Results depict that in spite of less flexibility this tool still has better accuracy than other packet pair tools. Asymptotic Dispersion Rate (ADR) representing the utilization rate of all the links is an important metric for ensuring QoS guaranteed by path. However, ADR is not the $\mathrm{ABW}$ as considered by the previous work.

- $\quad$ Bprobe [29]

This tool measures the speed of slowest link thereby providing base bandwidth of link between any two nodes within a network. The basis of this tool is to transmit from 
sender to recipient and back to sender a sequence of ICMP ECHO packets and measuring returning packets inter arrival time gaps. Throughout the multi-hop route, the packets travel links with different capacities, thereby affecting inter-packet arrival time gap. This time gap is inversely proportional to the capacity of various link's constructing the route. This time gap is enlarged while passing through least capacity link called "Bottleneck link". The returned packet at source reflects the speed of the least capacity link. The notion behind Bprobe tool is if two packets travelling together through least capacity link are queued as a pair at this link, the inter-packet arrival time spacing will be in proportion to the time taken by this least capacity link's router for processing the second packet of the pair. The base bandwidth of this least capacity link is given as follows:

$$
B B W=\frac{\rho \text { bytes }}{\text { gap seconds }}
$$

Where BBW represent the base bandwidth of a bottleneck link measured in bytes per second. And $\rho$ represent the size of the packet in bytes and gap represent the interarrival time of the packet in seconds.

- $\quad$ Sprobe [30]

This tool executes at the Sender side only to evaluate path's capacity. Under this tool, Sender transmits TCP SYN packet pairs and receives corresponding TCP RST packets from the remote host; thereby facilitating the packet pair dispersion calculations in forward path at the sender. If remote host runs a web or gnutella server, the reverse path capacity could also be evaluated using this tool by transferring a short file from the remote host and inspecting the dispersion of packet pairs sent by TCP during slow rate.

\subsubsection{End-to-End ABW Estimation Tools}

- Initial Gap Increasing (IGI) [31,32]

In this tool, $\mathrm{ABW}$ of a route within network is estimated by observing the input packet pair's time gap which in turn provides the packet pair's time gap relation with respect to competing traffic on the least capacity link. This tool within a one-hop network transmits packet trains with growing time gap and evaluates their corresponding relationship with the competing traffic on the least capacity link. Corresponding to every probe packet train, the variation between average output gap and input gap is observed, thus facilitating the ABW calculation as the difference between the evaluated bandwidths of competing traffic and least capacity link. Within one-hop networks IGI has minimal errors as it precisely evaluates the competing traffic amount on least capacity link router. However the accuracy is deteriorated in multi-hop networks with less competing traffic as light traffic induces only smaller number of probing gaps. For measurement of competing traffic, input packet time gap serves a significant role. Dynamically varying competing traffic is very important as packet pair gap can precisely evaluate the ABW.

- $\quad$ Pathchirp [36]

This active probing tool is based on the notion of "self-induced congestion". Unique feature of this tool is an exponential spaced chirp probing train. At the receiver side, statistical analysis is performed over the queuing delays i.e. packets inter-arrival times corresponding to the probe packets transmitted from sender for the evaluation of path's ABW. Till the time probing rate is smaller than the ABW of route, no queuing delays are observed, but the moment it increases more than route's ABW queuing starts thereby increasing transfer time. Thus, this probing rate at which queuing starts indicating congestion of network is equivalent to the $\mathrm{ABW}$ of route. Pathchirp tool works well for one-hop as well as multi-hop routes.

- $\quad$ Pathload [35]

An original end-to-end ABW evaluation technique known as "Self-Loading Periodic Streams (SLoPS)" is the basis for this tool. As per SLoPS, the one way periodic stream delays increases if stream rate is more than ABW. Pathload comprise of two distinct processes, one at sender and other at recipient. Periodic packet streams are 
generated using UDP-User Datagram Protocol while control channel in-between two end terminals is set up by TCP connection. Each transmitting packet is time stamped by sender process corresponding to which one way time delay of every packet is evaluated by recipient terminal which differs from actual value by certain offset. As per this algorithm, the packet stream rate denoted by $\mathrm{R}$ should be equal to ratio of packet size $\mathrm{L}$ to transmission duration $\mathrm{T}$ i.e. $\mathrm{R}=\mathrm{L} / \mathrm{T}$ in order to evaluate the maximum $A B W$. If measured value of $R$ is lesser than the $L / T$ value, then interspacing time $\mathrm{T}$ is decreased to $\mathrm{L} / \mathrm{R}$. Thus, the ABW measured by this tool is $\mathrm{L} / \mathrm{T}$ which is nothing but the maximum rate that Pathload can produce. As this tool imposes insignificant impact on network overhead and delay, this tool is nonintrusive..

- $\quad$ Cprobe [29]

This tool evaluates the ABW of route. Under this concept, short streams of echo packets are transmitted to the recipient. The throughput achieved by probe packets is equivalent to the $\mathrm{ABW}$ denoted by $\mathrm{B}_{\text {avail }}$ is then evaluated as the total amount of bytes sent divided by the total time consumed from first till last packet reception.

Further, the bottleneck link's utilization rate ( $\left.\mu_{\text {probe }}\right)$ can be determined by dividing the measured ABW (Bavail) with bottleneck link's speed (Bbls). i.e.

$$
\mu_{\text {probe }}=\frac{B_{\text {avail }}}{B_{\text {bls }}}
$$

Extreme inter-arrival measurement on both higher and lower end are dropped for improving the precision of measured $\mathrm{ABW}$.

\subsubsection{Bulk-Transfer Capacity Estimation Tools}

- $\quad$ TReno [34]

TReno is a bulk transfer capacity measurement tool of a network path. TReno deals with sending ICMP or UDP "data packets" that generate ICMP "ACKs" from the receiver of the data packets. TReno is one end point tool, means that user needs to control only the sending host not the receiver host. Receiver is an arbitrary host connected over an arbitrary network path. However, this is also a disadvantage of TReno because user has to infer the behaviour of the receiver. This will impact on the congestion window. The inability of TReno to differentiate between data packet losses from source to target and ACK losses from target to source is another disadvantage of this tool. The concept of assuming inflated rate of losses very higher than the TCP sender's observed rate of losses imposes adverse effect on its performance, thereby providing imprecise bulk transfer capacity evaluation. TReno implements an advanced loss rate recovery algorithm based on the selective acknowledgments (SACKs). So it is difficult to compare the performance of TCP which did not support SACK and TReno. TReno use static time of 2 seconds for the retransmission timeout (RTO). Finally TReno does not model an advertised window size.

- Cap [33]

Cap is another bulk transfer capacity measurement tool. Unlike TReno, It is two end point tool means user controls Source node as well as the Target node. This tool utilizes two different programs- first one is sender program (cap) and the second one is receiver program (capd). For data purpose, this tool transmits "UDP" packets, while for acknowledgment it utilizes "ACK" packets. The ACK packet not only includes its sequence number called as "ACK number" but also consist of an exclusive identifier corresponding to its concerned data packet in order to emulate TCP behavior. Large numbers of command line options are used to control each program.

\section{Passive Bandwidth Estimation Techniques}

Researches are now inclining towards passive bandwidth estimation techniques due to the limitations of active techniques. Passive techniques also termed as calculation based techniques measures the local consumption of bandwidth in order to evaluate the ABW. 
These techniques are more preferred choice since they do not induce any additional overhead to the network traffic due to the absence of any probing packet. Here, the channel is passively sensed for a pre-defined interval of time with respect to certain sensing threshold depending on the intended region to be covered for any communication happening within that range without interfering with those existing communications, thereby measuring the proportion of time for which medium is sensed busy which in turn provides the medium idle sensed proportion. This idle-period is further used in conjunction with channel maximum capacity in order to evaluate the ABW of a node. In IEEE802.11 $\mathrm{MAC}$, carrier-sensing range is utilized to sense medium. Within the carrier-sensing range of a node if any node is communicating to other node /nodes then the concerned node will sense the medium busy otherwise idle. In case the medium is sensed busy then the sensing node will wait for further channel access for a period of time called NAV (Network Allocation Vector) which is set equal to the duration of time required for the sensed communication happening in the vicinity as mentioned in its data packet header. Once this NAV value is decremented till current clock time then the concerned node will try to sense the medium again in order to gain channel access.

In spite of its simplicity, it suffered from mobility problem that a broken route couldn't be identified until next data transmission. Most of the researches overcome this problem by introducing HELLO packets for exchanging local info at regular predefined intervals in their routing protocols. Due to infrequent exchange and comparatively very small size of Hello packets the passive techniques are considered Non-Intrusive.

In further sub-sections, we briefly enlightened the various passive estimation techniques like QoS-AODV [37], BRuIT [38], CACP [39], AAC [40], ABE [41], IAB [42], RABE [43], ABE_MM [44], and DLI-ABE [45] and BECIT [46] used for wireless communications.

Table 1. Summary of ABW estimation techniques

\begin{tabular}{|c|c|c|c|}
\hline $\begin{array}{l}\text { Name of } \\
\text { Techniqu } \\
\text { es }\end{array}$ & $\begin{array}{c}\text { Accurac } \\
\mathbf{y}\end{array}$ & $\begin{array}{c}\text { Overhe } \\
\text { ad }\end{array}$ & Focused challenges \\
\hline $\begin{array}{l}\text { QoS- } \\
\text { AODV }\end{array}$ & Medium & None & $\begin{array}{l}\text { This technique incorporates QoS-aware route finding } \\
\text { algorithm, Explores different ways of ABW } \\
\text { estimation and Route Maintenance. }\end{array}$ \\
\hline BRuIT & Medium & Low & $\begin{array}{l}\text { Deals with problem of Interference due to distant } \\
\text { nodes, BRuIT provides to the nodes information about } \\
\text { their neighbours, performs admission control on each } \\
\text { bandwidth reservation request. }\end{array}$ \\
\hline CACP & Medium & Higher & $\begin{array}{l}\text { 1. Prediction of ABW. } \\
\text { i) Calculation of Local ABW. } \\
\text { ii) Prediction of c-neighbourhood ABW. } \\
\text { 2. Quantitative estimation of the bandwidth required } \\
\text { to start new flow within the network. }\end{array}$ \\
\hline AAC & Medium & None & $\begin{array}{l}\text { AAC consider four main issues: } \\
\text { i) Carrier Sensing } \\
\text { ii) Inter-Flow interference } \\
\text { iii) Intra-Flow interference } \\
\text { iv) Threatening mobility }\end{array}$ \\
\hline $\mathrm{ABE}$ & Low & None & $\begin{array}{l}\text { ABE taken into account the Node's Emission } \\
\text { capabilities, Probabilistic approach for } \\
\text { synchronization between idle period sensed by source } \\
\text { and recipient, Incorporated the probability of } \\
\text { collisions and backoff idle time as a result of } \\
\text { collisions. }\end{array}$ \\
\hline
\end{tabular}




\begin{tabular}{|c|l|l|l|}
\hline IAB & High & None & $\begin{array}{l}\text { IAB considered the synchronization between idle } \\
\text { period sensed by source and recipient for actual } \\
\text { workload. }\end{array}$ \\
\hline RABE & High & None & $\begin{array}{l}\text { RABE taken into account the bandwidth wasted due to } \\
\text { extra waiting times and average retransmission } \\
\text { attempt. }\end{array}$ \\
\hline ABE_MM & High & None & $\begin{array}{l}\text { Estimation of ABW by taken into account the } \\
\text { Mobility criterion in ad hoc network. }\end{array}$ \\
\hline DLI-ABE & High & Low & $\begin{array}{l}\text { 1. Computation of synchronization between idle } \\
\text { period sensed by source and recipient on the basis } \\
\text { on three states: BUSY, SENSE BUSY, and IDLE. } \\
\text { 2. Introduction of distributed Lagrange interpolation } \\
\text { for evaluating probability of collisions. } \\
\text { 3. Estimation of bandwidth lost due to extra waiting } \\
\text { time backoff and inter frame spacing like } \\
\text { DIFS,SIFS etc. }\end{array}$ \\
\hline BECIT & High & None & $\begin{array}{l}\text { ABW estimation using Cognitive Agent (CA) based } \\
\text { on probability of collisions, synchronization between } \\
\text { idle periods of source and recipient and randomized } \\
\text { waiting times. }\end{array}$ \\
\hline
\end{tabular}

\subsection{QoS Enabled routing in Mobile Ad hoc Networks (QoS-AODV) [37]}

This routing protocol employs real time "admission control scheme" and "feedback scheme" for ensuring QoS requirements. It calculates bandwidth availability at each node which is further used to control the network traffic. For evaluating the remaining bandwidth at each node this protocol utilizes "Listen bandwidth estimation method" and "Hello bandwidth estimation method". QoS-AODV calculates and broadcasts to one-hop neighbors using Hello message the ratio of packets received with respect to the number of transmitted packets and terms it as BWER (Bandwidth Efficiency Ratio). Out of the BWER values received through Hello messages from all its one-hop neighbors' each node checks for the least BWER value and multiplies it with channel maximum capacity in order to obtain its ABW. Statistical outcomes depict that the packet delivery ratio grows rapidly while network delay and power wastage reduces prominently. Hello messages add extra overhead as these are sent after every second.

\subsection{Bandwidth Reservation under InTerferences influence (BRuIT) [38]}

This distributed protocol reserves bandwidth by focusing on the existence of interference from far transmissions in wireless ad hoc networks. BRuIT considers that two terminals can be still fighting for the same resources even if they are not within each other's direct transmission range. Carrier sensing range is assumed approximately twice the transmission range. This technique has the assumption that full network's information is available at each and every terminal. As the very first task, BRuIT determines for each node a clique of interfering nodes. Each node at predetermined regular interval broadcasts its address and bandwidth consumption for existing flows in the form of Hello message to their one-hop neighbors thereby based on this received information enabling the nodes to evaluate the remaining bandwidth that it can use for admitting new flows in the network. Therefore, the admission of new flows can be controlled on the basis of ABW. BRuIT performs the Filtering of flows which control and avoid the congestion in the network. BRuIT is used mainly in wireless ad hoc network in which nodes has whole knowledge of interference. Frequency and size of hello packet influence the signaling overhead. BRuIT allows accurate bandwidth reservation by transmitting information on the load of the radio medium. 


\subsection{Contention - Aware Admission Control Protocol (CACP)}

This protocol supports QoS by controlling new flow's admission while sustaining end-to-end routes within network. Allocating bandwidth to a node is determined considering two parameters- (i) the "c-neighborhood ABW" calculated as the least value of local ABWs of all nodes within its carrier-sensing range i.e. c-neighborhood. (ii) The contention effect caused by multiple nodes within a route affecting the same resources. Admitting a new flow is controlled by comparing the new flow bandwidth requirements admission with respect to the ABW which instead of a local concept is the minimum of node's on local ABW and its c-neighborhood ABW. The "c-neighborhood ABW" is defined as the highest value of bandwidth resource that a node can utilize to initiate a new communication without disturbing the performance of already existing flows in its cneighborhood. Whereas, while sensing within its carrier sensing range, the idle time proportion perceived by a node with respect to total observation time further multiplied by the channel maximum capacity provides the "Local ABW" of that node which indicates its own unused bandwidth. Thus, in order to admit a new flow a node must possess ample local as well as c-neighborhood ABW. CACP proposed three different ways to evaluate the c-neighborhood ABW:

$>$ CACP-Multihop: In this methodology, all Nodes broadcast Hello message to their single-hop all neighbors i.e. all neighbors in their transmission range and further the receivers of these Hello message forward it to their all single hop neighbors. This Hello packet transmission happens till k-hops. Typical value suggested by CACP-Multihop is 2 assuming all the nodes in a node's carrier sensing range are covered by 2 hops. The Hello packet sent by initial nodes contain their own local ABW information, the moment it is further broadcast by trans-receiver to next hop they also add their own Local ABW information. Thus final receiving node at khop distance has the information of its k-hop neighbor's local ABW along with its on Local ABW. The least value of self and c-neighborhood Local ABW is then compared with the new flow bandwidth requirement to check the feasibility of new flow while maintaining QoS.

$>$ CACP-Power: Instead of propagating the Hello message to 2-hops to gather the information of c-neighbor's local ABW, the power to transmit the Hello message is increased at transmitter level such that it can cover directly all neighbors within carrier sensing range. The result obtained through this technique is very precise as it covers only but all the nodes within carrier sensing range. As regulations restrict the maximum transmission power that could be employed, this technique is generally avoided except where some additional measures like "transmission power control" (TPC) are employed for normal transmissions.

$>$ CACP-CS: Under this technique, "neighbor carrier sensing threshold" is defined which is set much lesser than the threshold for sensing c-neighbors. The notion behind this threshold is to sense the possible transmission of not only its own cneighbors but also the c-neighbors of its c-neighbors which may be affecting the local ABW of its c-neighbors. The area covered under this range is termed as "Neighbor-carrier-sensing Range". It may be noted that this neighbor carrier sensing threshold is used to just sense the possible communication but not to clearly interpret the communication which requires signal strength above SNIR (Signal to Noise plus Interference Ratio) threshold. A node sensing the medium having signal strength greater than this "neighbor carrier sensing threshold" assumes the channel as busy otherwise idle. During a certain time frame the proportion of idle channel sensed duration is proposed as the c-neighborhood ABW.

Statistical results depict that CACP is an admission control protocol which provides QoS guarantee in applications in terms of not only bandwidth but also delay and jitter.

\subsection{Adaptive Admission Control (AAC) [40]}

This admission control protocol by utilizing the QoS routing across layers asserts accurate evaluation of end-to-end availability of bandwidth. AAC considers four main 
issues when performing admission control which are: carrier sensing, Inter-flow interference, intra-flow interference $\&$ mobility. This protocol for each node assumes the clique of probable contending nodes equivalent to a single node. It perceive the medium idle period duration which could be utilized as a frame to transmit corresponding size data. Each node evaluates its own ABW and broadcast this info to one-hop neighbors, thereby facilitating the single-hop link's ABW. While measuring the channel occupancy, Far-flung transmissions and collisions are also taken into consideration. As per AAC, for a link between two nodes i.e. one sender (s) and one receiver (r) having local $A B W s A_{s}$ and $A B_{r}$ respectively, the $\mathrm{ABW}$ this link could be evaluated as:

$$
\mathrm{AB}_{(\mathrm{s}, \mathrm{r})}=\min \left\{\mathrm{AB}_{\mathrm{s}}, \mathrm{AB}_{\mathrm{r}}\right\}
$$

The results obtained by employing this protocol are over-estimated as it is based on the assumption that both nodes constituting the particular link have overlapping idle sensing time frames. AAC could be employed for managing radio channel and providing QoS in MANET's.

\subsection{ABW Estimation (ABE) [41]}

It estimates the availability of bandwidth between each link without disturbing any already exiting flows in the network. ABE focus four main challenges for calculating the ABW between each link which are: Evaluating the capabilities of a node for transmission, synchronization between idle periods sensed by source and recipient, prospect of collisions, backoff idle time as a result of collisions. Node's idle duration is measured as the total time for which there is no transmission from that node itself, as well as it is not sensing the channel occupied by any other communication in its vicinity. ABE presented a probabilistic approach in order to compute the source and recipient synchronization. Further, the probability of collisions is computed by utilizing the probability of collisions ( $\mathrm{P}_{\text {Hello }}$ ) of Hello packets used in routing protocol and developing a relationship equation for corresponding collision probability $\left(\mathrm{P}_{\mathrm{M}}\right)$ of data packets of size $\mathrm{m}$ bits. The proposed relationship $\mathrm{f}(\mathrm{m})$ which is further interpolated in ABE techniques using Lagrange's Interpolation polynomial is as follows:

$$
\mathrm{P}_{\mathrm{M}}=\text { PHELLO }^{*} \mathrm{f}(\mathrm{m})
$$

ABE assumes that both nodes constituting the links have independent channel occupancy. As per ABE, the bandwidth available to a link could be computed by following equation:

$$
A B W=(1-K) \times\left(1-P_{c}\right) \times\left(\left(\frac{T_{s}}{T}\right),\left(\frac{T_{r}}{T}\right)\right) \times C
$$

Where, $\mathrm{P}_{\mathrm{c}}$ denotes the probability of collision of data packet, $\mathrm{K}$ signifies the bandwidth wasted due to back-off concept and waiting, $\mathrm{T}_{\mathrm{s}}$ and $\mathrm{T}_{\mathrm{r}}$ represents the time for which medium is perceived idle by source and recipient respectively within a specified observation duration T, and C represents is the channel's maximum capacity. Simulations depicts that by utilizing $\mathrm{ABE}$ technique, a throughput more than $95 \%$ could be achieved. The non-consideration of control overheads may induce inaccuracy in ABE's estimation. For multimedia applications demanding higher accuracy this technique is best suited.

\subsection{Improved $A B W(I A B)[42]$}

This technique is a modification of the previously explained $\mathrm{ABE}$ techniques as it not only considers the source and recipient's idle time's synchronization but also incorporates the dependency between these time frames. IAB suggest that in addition to "Idle" and "Busy" sensing stats there is another sensing state termed as "Sense Busy" state. As per $\mathrm{IAB}$, if a node it-self transmitting or receiving then that state is "Busy state" but if node is sensing medium busy not because of its own communication then that state is "Sense Busy state". If the node is neither "Busy" nor "Sense busy" then it is considered as "Idle State". This differentiation between "Busy" and "Sense Busy" states impacts the idle time period 
and thus the overall ABW of the link is influenced. Simulations clearly demonstrate that IAB has an improved accuracy over the previously existing techniques in terms of bandwidth estimation of a link. Applications where the bandwidth is major limitation employ this technique afore accomplishment of QoS conscious functions like QoS routing, controlling new flows admissions and managing the existing flows.

\subsection{Retransmission-based ABW Estimation (RABE) [43]}

This technique takes into picture the losses due to retransmission in terms of amount of bandwidth wasted because of additional waiting duration and channel occupancy. It incorporates the retransmission attempts average while evaluating bandwidth availability. $\mathrm{ABE}$ technique focuses on the parameter such as $\mathrm{ABW}$ at a node, idle period synchronization, collision probability and average backoff. But none of the existing technique taken into account the bandwidth lost due to retransmission attempt. In 802.11 DCF, whenever a collision occurs, the collided packet is retransmitted till its maximum retry limit is exhausted and then discards the packet. This retransmission as well as discarding the packet remarkably impacts the bandwidth availability. Statistical data depicts that RABE accomplish a "mean error ratio" of $17 \%$ as compared to real measured value. Accuracy achieved by this technique could be as high as twice that of $\mathrm{ABE}$ and 10 times that is achieved by IAB.

\subsection{Distributed Lagrange Interpolation based ABW Estimation (DLI-ABE)[47]}

This technique is further improvement in [41] and [42]. DLI-ABE estimates the ABW based on synchronization between idle time frames of source and recipient; incorporating distributed Lagrange interpolation polynomial for evaluating probability of collisions and arbitrary waiting durations. DLI-ABE focus is on three main points which are 1) in order to prohibit a node from gorging the bandwidth resource because of total overlap and never over periods, the authentic medium utilization and degree of collision is employed for measuring the synchronization of idle time frames of source and recipient. 2) Before transmitting actual Data packets, each node individually estimates the probability of collision by utilizing "distributed Lagrange Interpolation polynomials". 3) Calculation of arbitrary wait timings out of the backoff duration, Short Inter-frame spacing time, Distributed Inter-frame spacing time, RTS/CTS time consumed and delay time of the acknowledgement received.

As a result of employing distributed Lagrange interpolation polynomial at individual nodes before their actual data packets transmission, this technique gains better accuracy than $\mathrm{ABE}$ technique. $\mathrm{ABE}$ also uses same polynomial without paying attention to network topology and interfering nodes. The increase in number of interfering nodes escalates the probability of collisions. Thus still deploying the same polynomial even after network topology change may induce inaccuracy in measuring probability of collision and thereby affecting ABW evaluation results. Experiments prove that DLI-ABE could achieve $19.99 \%$ higher accuracy than ABE.

\subsection{ABW Estimation with Mobility Management (ABE_MM)[44]}

Due to the existing ABE technique's drawback to efficiently deal with node's mobility, here a mathematical approach is proposed to integrate node's mobility factor " $M$ " with already existing ABE technique's results. Proposed new equation by this technique is as follows:

$$
A B E_{M M}=(1-k) \cdot(1-p) \cdot b_{(s, r)} \cdot M
$$

Where, $\mathrm{K}$ is the factor for bandwidth wasted in backoff mechanism, $\mathrm{p}$ is the probability of data packet collision, $b_{(s, r)}$ is the product of channel capacity multiplied with idle time proportion out of total observation period of two nodes constituting the link for which the final bandwidth availability ABEMM is calculated.

The Mobility factor " $\mathrm{M}$ " is calculated utilizing the basic principles of communication, by observing the signal strength (RSS) measured by recipient node corresponding to 
source's sending signal strength (SSS) as a function of varying distance "d" between them. For a free-space propagation model, the RSS could be determined as per following equation:

$$
\mathrm{RSS}=\operatorname{SSS} .(\lambda / 4 \pi \mathrm{d})^{2} . \mathrm{GT}_{\mathrm{T}} . \mathrm{GR}_{\mathrm{R}}
$$

Where, $\lambda$ represents the wavelength of the medium, $\mathrm{G}_{T}$ and $\mathrm{G}_{\mathrm{R}}$ are the gain accessed by transmitting and receiving antenna's respectively. Thus the received signal strength is inversely proportional to distance " $\mathrm{d}$ " between the two nodes.

At present measurement time $\left(t_{1}\right)$ the received signal strength is measured $\left(\mathrm{RSS}_{1}\right)$ corresponding to distance $\mathrm{d}_{1}$ between them and compared with the value $\mathrm{RSS}_{2}$ measured at previous measurement interval $\left(\mathrm{t}_{2}\right)$ corresponding to its previous distance $\mathrm{d}_{2}$. Further, the relative speed (SP) of recipient node with respect to source node is calculated as:

$$
\mathrm{SP}=\left(\mathrm{d}_{1}-\mathrm{d}_{2}\right) /\left(\mathrm{t}_{1}-\mathrm{t}_{2}\right)
$$

Based on the speed calculated, the time $\left(T_{j}\right)$ required by this mobile recipient node to go out of transmission range of transmitter could be evaluated by simple mathematical expression as:

$$
\mathrm{T}_{\mathrm{j}}=\left(\mathrm{C}_{\mathrm{d}}-\mathrm{d}\right) / \mathrm{SP}
$$

Where, $C_{d}$ is the transmission range of transmitter and $d$ is present distance of recipient from transmitter. Further the mobility factor " $\mathrm{M}$ " is evaluated as:

$$
M=\left(T_{j}-T_{n-1}\right) / \Delta
$$

Where, $T_{n-1}$ denotes the present time at which bandwidth is evaluated using ABE formula, $\mathrm{T}_{\mathrm{j}}$ is the moment where the link will breaks (i.e. $\mathrm{d}>\mathrm{C}_{\mathrm{d}}$ ) and $\Delta$ represents the measurement period duration. Experiments prove that the results of $A B E$ MM are much more precise than $\mathrm{ABE}$.

\subsection{ABW Estimation Using Probability of Collisions, Idle time frames Concurrence and Arbitrary waiting Time in MANET's: Cognitive Agent Based Approach (BECIT) [46]}

BECIT further extends the work present in [47] by utilizing intellectual software program called "Cognitive Agent (CA)" for evaluating the ABW in MANETs. Cognitive agent works as wisely as human being in decision making. CA further investigates the task based on three indices: (i) Belief, (ii) Desire and (iii) Intention. BECIT utilizing the cognitive agent on the behalf of user performs the evaluation of ABW keeping in mind the idle time frame's synchronization at source and recipient prior to actual data transmission as well as the arbitrary waiting duration and probability of collisions. It employs 2 modules to evaluate the ABW: "Pre-Analysis module" and "AB-Estimation module". The 2 modules operate at same norm of BDI (Belief, Desire and Intention) model through software program i.e. cognitive agent. The "Pre-analysis module" evaluates the synchronization of idle time frames of source and recipient as well as the probability of collisions by utilizing the cognition feature. Whereas, the "AB-estimation module" evaluates the ABW and also sustains the results of Pre-analysis module i.e. idle time frames and probability of collisions. Results show that BECIT technique could be $30 \%$ more accurate as compared to $\mathrm{ABE}$ technique for evaluating bandwidth availability.

\section{Model Based Techniques (MBT)}

Passive techniques that are described in previous section are not much adequate in estimating the ABW, because they can't predict the result set after the admission of new flow in the network. They just consider that on acceptance of new flow network parameters get changed and which will effect on the real ABW. So future result set can't be predicted by active/passive bandwidth estimation techniques. Analytical techniques are much more suited for network performance analysis. It is a challenging task to build a mathematical model for multiple-hops radio network. Within wireless network by utilizing the DCF operations, there have been proposed some analytical prototypes. Analytical techniques are broadly classified into three main categories: saturated, unsaturated and semi-saturated. Saturated networks are those networks which is overloaded means every node has a packet ready to be sent. Unsaturated networks can be defined as lightly loaded network and node is not saturated. Semi-saturated networks lie in between saturated and un-saturated network, 
i.e. both saturated as well as un-saturated nodes exists in semi-saturated networks. Further sub-sections provide a brief overview of various Model Based Techniques as follows:

\subsection{Performance Analysis of the IEEE 802.11 Distributed Coordination Function[60]}

While considering the deterministic number of nodes and under idealistic network environment, this technique offers a modest and enormously precise analytical frame for evaluating the throughput achieved in 802.11 DCF protocol. Both, the "basic access scheme" employed as the data transmission mechanism in DCF as well as the channel access "RTS/CTS schemes" are treated with this analytic technique. Further, this technique is also employed for mixture of these two schemes by transmitting packets utilizing RTS/CTS scheme that are higher than certain limit value. Neglecting the presence of hidden nodes and capture effect, thereby assuming idealistic network scenario, this analytical technique mainly contributed towards saturation throughput assessment. Not only the nodes count is anticipated to be constant but also each node is considered to always have packets for transmission, i.e. the network is presumed to be saturated such that any node's transmission queue is never empty.

As a first step of this analysis, the "Markov model" is deployed to study the nature of single terminal thereby evaluating the "stationary probability $(\tau)$ " by which the node is transmitting packet within nonspecific time intervals. This evaluated probability is independent of the access scheme (basic or RTS/CTS) used. In next step, events that could happen within a generic time frame are studied in order to articulate the throughput in terms of computed value $\tau$ of these access two "basic" and "RTS/CTS" schemes as well as their amalgamation.

Results depict that factors like contention window least size and nodes count within the network intensely affect the "Basic access" scheme's outcome. Whereas, the "RTS/CTS" scheme's outcome are least bothered by these factors.

\subsection{Performance analysis of the IEEE 802.11 MAC protocol for wireless LANs[62]}

Neglecting the presence of hidden terminals and transmission slips, "Markov chain modelling" is employed for the performance assessment of IEEE802.11 DCF. This technique not only computes the throughput efficiency, but also assesses the average packet delay, likelihood of packet failure and the average packet drop interval in "Basic" as well as "RTS/CTS" channel access methods. This methodology considers network saturation such that for every terminal when a data packet is transmitted successfully another packet is always waiting at that transmitter. To incorporate this approach, the probability of collisions is presumed to be steady and is not dependent on historical packet collisions count. Under both "Basic" as well as "RTS/CTS" channel access methods, at the exhaustion of packet re-transmission limit, the parameters like throughput, average data packet lag and chances of packet failure are evaluated in order to access the performance of IEEE802.11 protocol. This analysis can be employed for both "basic" as well as "RTS/CTS" channel access schemes. This analysis's outcomes precisely project the performance of IEEE802.11 protocol as compared to the protocols which are considering continuous back-to-back data transmission without back-off mechanism.

Results depict the superiority of "RTS/CTS" scheme over the "Basic access" scheme when network size is bigger and data transfer rate are less.

\subsection{Model-based ABW Estimation for IEEE 802.11 Data Communications (MBE)[74]}

TCP/UDP based throughput models in wireless communications are employed for analytical evaluation of the bandwidth availability. MBE takes TCP and UDP traffic separately to estimates the bandwidth availability. Fast re-transmissions and concept of time-out are considered while evaluating the apex value of ABW of TCP link. Original TCP model get updated by considering probability of unsuccessful data packet delivery update, "round trip time" update and considering TCP model simultaneously with 802.11DCF model. MBE focus on three main points: (i) The IEEE802.11 WLAN features like transmission error, contention among neighboring nodes and re-transmission tries are 
integrated into the prevailing TCP throughput prototype in order to generate a new TCP model. (ii) IEEE802.11 network delay with UDP data transmission probability is utilized to create a new UDP throughput model. (iii) Under the presence of TCP and UDP traffic simultaneously in IEEE802.11 novel bandwidth estimation equation is derived. This technique neither employs probing traffic nor any alteration in MAC protocol.

Results show that MBE present lower overhead and higher estimation accuracy. The absence of probing traffic reduces induced overheads in bandwidth estimation. MBE model is used for multimedia application in wireless data communications.

\subsection{Model-based approach for ABW prediction in multi-hop wireless network[64]}

This absolute end to end bandwidth availability evaluation model offers a guaranteed value of throughput achievable to the applications requiring multi-hop wireless communications. It works under non-saturated environment keeping the consideration of interferences caused due to other flows existing in the vicinity, collision encountered, illeffect of hidden nodes, capture phenomenon and busy medium in multi-hop wireless communication networks. This model delivers guaranteed throughput by means of evaluating end-to-end bandwidth availability of a route consisting of various nodes within the network and controlling the new multimedia application's flow admission. Admitting a new flow is controlled by utilizing the "Binary search" algorithm and the region being searched is reduced to half each time thereby monitoring the apex capacity. Admission is granted to the new flow when the new flow's bandwidth requirement is lesser than this measured capacity, thus already existing flows in the network are not affected and QoS is maintained. This process get continues to search in the upper half and then the lower half. Relation between the ABW and the interference is not linear. The number of intermediate hopping count increase causes variations in bandwidth availability of the route under consideration. Applications like video-streaming, video-conferencing, network games etc. which require stringent bandwidth constraints prefers this technique due to guaranteed throughput availability.

\subsection{Performance of Reliable Transport protocol over IEEE 802.11 Wireless LAN: Analysis and Enhancement [58]}

Here the outcome of "reliable transport control" is improved over wireless local area networks. In IEEE802.11 WLAN instead of deploying existing DCF, a novel DCF+ scheme is introduced which enhances the results of reliable transport protocols over WLAN like TCP. The overall performance of already existing TCP over WLAN may suffer from collisions because of bi-directional flow i.e. Data packet from source to recipient and ACK from recipient to source. The full compatibility of DCF+ with DCF comfortably supports the coexistence of both schemes simultaneously such that even nodes not supporting DCF+ can smoothly exchange data with those supporting DCF. In both "Basic" as well as "RTS/CTS" schemes, the DCF+ access method, exchanges reverse direction control packets only after the original data exchange in the forward direction completed. The statistical experiments conclude the improvement in performance of reliable transport protocols in terms of good-put, fairness index and delay by deploying DCF + technique.

\subsection{Bandwidth Estimation IEEE 802.11 TCP Data Transmission[73]}

This all-inclusive ABW estimation model in IEEE802.11 WLAN's utilizing TCP data transmissions takes into account the parameters such as error during transmissions, retrial limits and connection establishment attempts. This model is deployed on application layer for evaluation of ABW. The throughput achieved by TCP is updated keeping these three steps into consideration: (i) Packet loss, (ii) Round-Trip-Time updating, and (iii) combining the TCP protocol with IEEE802.11 DCF architecture. In order to evaluate the ABW this model measures not only the size of data packet which is to be sent from source to recipient but also the feedback received from the recipient back to the source node, thereby calculating the packet loss rate corresponding to the number of clients. Because of its better accuracy in terms of $\mathrm{ABW}$ estimation over other existing techniques, this technique is 
employed for multimedia services in IEEE802.11 wireless communications requiring stringent QoS evaluation.

\subsection{Delay-Based Model (DBM)[72]}

This model keeps into consideration the three important components that are: 1) Scheme employed for sequencing of probing packets, 2) the characteristics of other flows in the vicinity creating network traffic, and 3) the protocol used to transmit packets in wireless communication network. Sequencing of probing packets could be further differentiated into three sub types based on the inert-arrival packet distribution: (i) a "Poisson Sequence" which provides an unbiased near-continuous detection, (ii) a "Periodic Sequence" that is relatively easier in implementation but concerned about the theoretical potency of a Poisson sequence, and (iii) an "Exponential Sequence" that captures the dependency of internet traffic in long time span, however the stochastic results are inaccurately analyzed. "SVC Probe" is used under this technique and the squared coefficient of deviation of departure time difference between two consecutive probing packets is measured, thereby utilizing this delay-variation to evaluate the ABW. Thus, the measured performance parameters of "Poisson" and "periodic" sequencing based active probing are used to deduce the ABW. Experimental results depict that the precision, losses, sturdiness etc. are improved by utilizing delay-based measurement schemes as compared to loss-based schemes. The deployment of delay-based model not only attains improved accuracy but also reduces probing time and overheads as compared with Pathload model.

\section{Conclusion}

Under a wireless communication networking scenario $\mathrm{ABW}$ evaluation is a challenging assignment as each host can't have the complete knowledge of the whole network and further the mobility of nodes induces complexity in terms frequent link variations. Bandwidth is a limited resource and knowledge of ABW within a network is indeed required for improving of quality of service. This paper described the bandwidth estimation techniques which are found in the literature. These techniques are: Active probing bandwidth estimation, Passive bandwidth estimation techniques and Model based techniques. For each category, an exhaustive survey of ABW evaluation techniques is provided. The performance indices like accuracy, overhead and delay are explored for these distinct methodologies in order to conclude their advantages and drawbacks. Till now, no bandwidth estimation technique is commercialized. This paper can prove to be very beneficial for those researchers who are working in this area. 


\section{REFERENCES}

[1] D. Helen, and D.Arivazhagan, "Applications, Advantages and Challenges of Ad hoc Networks", in Journal of Academia \& Industrial Research, Jan.2014, vol.2, issue.8.

[2] AleksiPenttinem, "Research on Ad-Hoc networking: Current Activity and Future Directions", in Networking Laboratory, Helsinski University of Technology, in FIN02015 HUT, Finland.

[3] Pravin Ghosekar, Girishkatkar, Dr. PradipGhorpade, "Mobile Ad Hoc Networking: Imperatives and Challenges", in International journal of Computer Applications on "Mobile Ad hoc networks MANETs", 2010.

[4] Xueyuan Su, Sammy Chan, Jonathan H.Manton, "Bandwidth Allocation in Wireless Ad Hoc Networks:Challenges and Prospects", in IEEE Communications Magazine, Jan.2010,vol.48, no.1.

[5] Jeroen Hoebeke, Ingrid Moerman, Bart Dhoedt and Piet Demeester, "An Overview of Mobile ad Hoc Networks: Applications and Challenges", in Journal of the Communications Networks, 2004, vol.3, no.3, pp.60-66.

[6] Prasad, R.S, Murray, M., Dovrolis, C.Claffy, K.C., "Bandwidth Estimation: Metrics, Measurement Techniques and Tools", in Journal IEEE Networks, Nov.2003, vol.17, no.16, pp.27-35.

[7] Pan Li, Yuguang Fang, Jie Li, "Throughput, Delay, and Mobility in Wireless Ad Hoc Networks", in IEEE INFOCOM, 2010, pp.1-9.

[8] Macro A. Alzate, Nestor M. Pena and Miguel A. Labrador, "Capacity, Bandwidth, and Available Bandwidth in Wireless Ad Hoc Network: Definitions and Estimations", in Florida,USA.

[9] Dr.Ashokkoujalagi, "Bandwidth Estimation for IEEE 802.11 Based Ad Hoc Networks", in International journal of Thesis project \& Dissertation, Sept.2014, vol.1.2, no.3, pp: 1-25.

[10] Qiang Ni, lamiaRomdhani, Thierry Turletti,“A Survey of QoS Enhancements for IEEE 802.11 Wireless LAN", in Journal of Wireless Communications and Mobile Computing, 2004,vol.4, issue 5,pp. 547-566.

[11] P.Chatzimisios, A.C.Boucouvalas and V.Vitas, "Performance Analysis of IEEE 802.11 MAC protocol for Wireless LANs", in International journal of Computer Science, June 2005.

[12] Janani J, O.SGnanapraksi, Dr.P.Varalakshmi, "A Survey on Available Bandwidth Estimation in Mobile Ad hoc Networks ", in International Journal for Advance Research in Engineering \& Technology, 2014,vol.,no.,pp.ISSN-2320-6820.

[13] Ningning Hu, Peter Steenkiste, "Evaluation and Characterization of Available Bandwidth Probing Techniques", in IEEE Journal on Selected Areas in Communications, Aug.2003, vol.21, no.6, pp.879-894.

[14] Cheikh Sarr, Claude Chaudet, Guillaume Chelius, and Isabelle Guerin Lassous , “ Bandwidth Estimation for IEEE 802.11 Based Ad hoc Networks" in IEEE transactions on Mobile Ad Hoc Networks, Oct.2008, vol.7,no.10,pp.1228-1241.

[15] K.Mohideen, VahithaBanu, "Discussion on Improving Quality of Service Through Available Bandwidth Estimation in Mobile Ad hoc networks", in International Journal of Computer Applications, Dec.2010,vol.11, no 8.

[16] Liang qin and Thomas Kunz , " Survey on Mobile Ad hoc Network Routing Protocols and Cross - Layer Design", in Carleton University,System and Computer Engineering, Aug.2004, vol.4.

[17] Neeraj Gupta , "Discussion on Impact of Carrier Sense Range on Available Bandwidth for IEEE 802.11 based Ad Hoc Networks", in CCIS 250 Springer,pp.274277.

[18] Turrubiartes, M., Torres, D., Angulo, M., \& Munoz, D. , "Analysis of IP network path capacity estimation using a variable packet size method", in 5th international conference on electronics, communications and computers, 2005, pp. 177-182.

[19] Selin, P., Hasegawa, K., \& Obara, H., “Available bandwidth measurement technique using impulsive packet probing for monitoring end-to-end service quality on the internet", in 17th Asia-Pacific conference on communications ,2011,pp. 518-523. 
[20] Obara, H., Koseki, S., \& Selin, P., "Packet train pair: A fast and efficient technique for measuring available bandwidth in the internet", in SICE annual conference, 2012, pp. 1833-1836.

[21] Xiao, Y., Chen, S., Li, X., \& Li, Y., "A new available bandwidth measurement method based on self-loading periodic streams", in Wireless Communications, Networking and Mobile Computing, WiCom 2007, pp.1904-1907.

[22] Jain, M., "End-to-end available bandwidth: Measurement methodology, dynamics, and relation with TCP throughput", in IEEE/ACM Transactions on Networking, 2003, vol.11, no.4, pp.537-549.

[23] Downey, A. B, "Using pathchar to estimate internet link characteristics", in $A C M$ SIGCOMM Computer Communication Review, 1999, vol. 29, no. 4, pp.241-250.

[24] Melander, B., Bjorkman, M., \& Gunningberg, P., "A new end-to-end probing and analysis method for estimating bandwidth bottlenecks ", in IEEE global telecommunications conference vol. 1, pp. 415-420.

[25] Downey, A. B. (1999). Clink: A tool for estimating internet link characteristics. http://rocky.wellesley.edu/downey/clink/

[26] Mah, B.A.(2001)Pchar: A tool for measuring internet path characteristics. http://www.employees.org/bmah/Software/pchar/

[27] Kevin Lai, Mary Baker, "Nettimer: A Tool for Measuring Bottleneck Link Bandwidth", in Proceedings of USENIX symposium internet technologies and sytems,Jan.2001,vol. 3, pp. 123-134.

[28] Lai, K., \& Baker, M. "Measuring link bandwidths using a deterministic model of packet delay", in ACM SIGCOMM Computer Communications Review, 2000, vol.30, no.4, pp. 283-294.

[29] Carter, Robert L., "Measuring bottleneck link speed in packet-switched networks", in Journal of Performance Evaluation, 1996, pp. 297-318.

[30] Zhou, H., "Measuring available bandwidth for smart cyber-physical applications", in Tsinghua Science and Technology, 2011, vol.16, no.6, pp.601-610.

[31] Hu, N., \& Steenkiste, P.," Evaluation and characterization of available bandwidth probing techniques", in IEEE journal on selected Areas in Communications, 2003, vol.2, no.6, pp.879-894.

[32] Hu, N., \& Steenkiste, P., "Estimating available bandwidth using packet pair probing", in CARNEGIE-MELLON UNIV PITTSBURGH PA SCHOOL OF COMPUTER SCIENCE, 2002.

[33] Mark Allman, "Measuring End-to-End Bulk Transfer Capacity", in ACM SIGCOMM internet measurement workshop, 2001.

[34] Mathis, M., \& Mahdavi, J., "Diagnosing internet congestion with a transport layer performance tool", in Proceedings of INET at Montreal, Quebec, Canada, 1996.

[35] Jain, M., \& Dovrolis, C., "Pathload: A measurement tool for end-to-end available bandwidth", in Proceedings of passive and active measurements workshop, 2002, pp. $14-25$.

[36] Vinay j. Riberio, Rudolf H. Riedi, Ricard G.Baraniuk, Jiri Navratil, C.Les, "PathChirp: Efficient Available Bandwidth Estimation for Network Paths", in Proceedings of $4^{\text {th }}$ passive active measurement workshop, 2003.

[37] R.de Renesse, V. Friderikos and H.Aghvami, "QoS enabled routing in mobile ad hoc networks", in Fifth IEEE international conference on $3 G$ mobile communications technologies, 2004, vol.1, no. 4, pp.678-682.

[38] Claude Chaudet, Isabelle Guerin Lassous, "BRuIT: Bandwidth Reservation under In Terferences influences", in Proceedings of European Wireless, 2002.

[39] YalingYang, Robin Kravets, "Contention- aware admission control for ad hoc networks ", in IEEE Transaction on Mobile Computing, July 2005, vol.4,no.4, pp.303-377.

[40] de Renesse, R., Ghassemian, M., Friderikos, V., \& Aghvami, A. H. , "Adaptive admission control for ad hoc and sensor networks providing quality of service", in Technical Report, King College London, 2005.

[41] Sarr, C., Chaudet, C., Chelius, G., \& Lassous, I. G., "Bandwidth estimation for IEEE 802.11-based ad hoc networks", in IEEE Transaction on Mobile Computing, 2008, vol.7, no.10, pp.1228-1241. 
[42] Haitao Zhao, Yong Xi, "Accurate available bandwidth estimation in IEEE 802.11 based ad hoc networks ", in Computer communication ,2009, vol.32,no.6, pp.10501057.

[43] Cheikh Sarr, Isabelle Guerin Lassous, Victor Moraru, "Retransmission - based bandwidth estimation in IEEE 802.11 - based multi-hop wireless networks", in $A C M$, 2011,vol.,no.,pp.978-1-4503-0898-4/11/10.

[44] Redouane Belbachir, ZoulikhaM.Mekkakia, Ali Kies , "Towards a New Approach in Available Bandwidth Measures on Mobile Ad Hoc Networks ", in IEEE Journal ,2011,vol.8, no.4.

[45] Shilpa Shashikant Chaudhari, Rajashekhar C. Biradar, "Collision Probability based Available Bandwidth Estimation in Mobile Ad Hoc Networks", in Fifth International Conference on the ICADIWT, Feb.2014, pp.244-249.

[46] Shilpa Shashikant Chaudhari, Rajashekhar C. Biradar, "Available Bandwidth Estimation Using Collision Probability, Idle Period Synchronization and Random Waiting Time in MANETs: Cognitive Agent Based Approach", in Springer Wireless Pers Commun., Dec 2015, vol. 85, no. 3, pp. 597-621.

[47] Haitao Zhao, Emiliano Garcia-Palacios, jibo Wei, and Yong Xi, “ Accurate Available Bandwidth Estimation in IEEE 802.11- based Ad Hoc Networks" , in Computer Communications ,2009, vol.32, no.6, pp.1050-1057.

[48] Lie Chen, Wendi B, Hienzelman, "QoS- Aware Routing Based on Bandwidth Estimation for Mobile Ad hoc Networks", in IEEE Journal on Selected Areas on Communications, March 2005, vol.23, no.3, and pp.561-572.

[49] Shilpa Shashikant Chaudhari, Rajashekar C. Biradar, "Survey of Bandwidth Estimation Techniques in Communication Networks", in Wireless Personal Communications, July 2015, vol. 83, no.2, pp.1425-1476 .

[50] C. Sarr, C.Chaudet, G.Chelius and I. Guerin Lassous, "A node - based available bandwidth evaluation in IEEE 802.11 ad hoc networks", in International journal of Parallel, Emergent and Distributed Systems, July 2005.

[51] K.Xu, K. Tang, r. Bagrodia, M. Gerla, and M. Bereschinsky, “ Adaptive Bandwidth Management and QoS Provisioning in large scale Ad hoc Networks" , in proc.Military Comm. Conf.( MILCOM '03), Oct.2003.

[52] Bob Melander, Mats Bjorkman , per Gunnignerg, "A New End to End probing and Analysis Method for Estimating Bandwidth Bottlenecks ", in IEEE global telecommunications conference, 2000, vol.1,pp.415-420.

[53] Qninghui Wang, Ansong Feng, "Available Bandwidth Estimation in IEEE 802.11 ad hoc networks", in HIS'09, Ninth International Conference on Hybrid Intelligent Systems, Aug.2009.

[54] Rajeshkumar Ramraj, "Bandwidth allocation and routing information for wireless mobile Ad hoc networks", in $7^{\text {th }}$ International Conference on Fuzzy Systems and Knowledge Discovery, Aug.2010, pp.978-1-4244-5934-6.

[55] Ningning $\mathrm{Hu}$, "Evaluation and Characterization of Available Bandwidth probing Techniques", in IEEE journal on Selected areas n Communications, Sept.2006, vol.21, no.6, pp.879-894.

[56] Nyambo Benny, JanseensGerrit, LamotteWim , “ Bandwidth Estimation in Wireless Ad hoc Networks ", in Journal of Ubiquitous \& Pervasive Networks ,2015, vol. 6, no.2,pp.19-26.

[57] Giuseppe Binachi, "Performance Analysis of the IEEE 802.11 Distributed Coordination Function", in IEEE Journal on selected Areas in communications, March2000, vol.18, no.3.

[58] S. Cheng, K. long, J.Ma, Y.Peng, and $\mathrm{H} . \mathrm{Wu}$, "Performance of reliable transport protocol over IEEE 802.11 wireless LAN: Analysis and enhancement", in IEEE conference on communication, 2002, vol 7, issue 6, pp.599-607.

[59] A.C.Boucouvalas, P.Chatzimisions and V.Vitas, "Performance analysis of IEEE 802.11 DCF in presence of transmission errors", in IEEE International Conference on Communications, June 2004, pp.3854-3858.

[60] Giuseppe Binachi,HeniaTinnirello,"Remarks on IEEE 802.11 DCF performance Analysis ", in IEEE Communications Letters , Aug.2005, vol.9. no.8. 
[61] Yang Xiao, " Performance Analysis of priority schemes for IEEE 802.11 and 802.11e wireless LANs", in IEEE Transactions on Wireless Communications ,July 2005,vol.4,no.4,pp.1506-1515.

[62] Anurag kumar, Eitan Altman, Daniele Miorandi, Munish Goyal, “ New Insight from a Fixed-point Analysis of Single Cell IEEE 802.11 WLANs", in IEEE Transactions on Networking ,2007,vol.15,no.3.

[63] Mohammad Hossein Manshaie, Jean-Pierre Hubaux, "Performance Analysis of the IEEE 802.11 Distributed Coordination Function: Binachi Model", in IEEE Mobile Networks, 2007.

[64] Haitao Zhao, Wang Shan, Wei Jibo "Model-based approach for available bandwidth prediction in multi-hop wireless networks," in Science China Information Sciences, June2011, vol.54, no.9.

[65] ChuanHeng Foh, jukiWirawan Tantra, "Comments on IEEE 802.11 Saturation throughput analysis with freezing of backoff counters", in IEEE communication Letters, Jan.2005, vol.9, no.2, pp.130-132.

[66] Haitao Wu, Yong peng, keeping Long, Shiduancheng, jian Ma, "Performance of Reliable Transport protocol over IEEE 802.11 wireless LAN: Analysis and Enhancement", in IEEE Computer and Communications Societies, June 2002, vol.02.

[67] IEEE. IEEE/IEE electronic library. http://ieeexplore.ieee.org.

[68] Pablo Brener," A Technical Tutorial on the IEEE 802.11 protocol" in Brezzecom Wireless Network, 1997.

[69] Haitao Zhao, Jibo Wei, Shan Wang and Yong Xi, "Available Bandwidth Estimation and prediction in Ad hoc Networks", in National University of Defence Technology.

[70] Ping Zhong, Xuemin Hong, Xiaofang Wu, Jianghong ShianhHuihuang Chen, "Comparison of pre-backoff and post backoff procedures for IEEE 802.11 distributed coordination function", in IEICE Electronics Express, 2011, vol.8, no.24, pp.20352040.

[71] EI-Sayad M.EI-Rabaie, Nancy A.AI-Shaer, “ A Survey on Ad Hoc Networks", in Conference, Nov.2016.

[72] Hei, X., Bensaou, B., \& Tsang, D. H. K., "Model - based end-to-end available bandwidth inference using queuing analysis", in Computer Networks, 2006, vol.50, no.12, pp.1916-1937.

[73] Yuan, Z., Venkataraman H., \& Muntean, G. (2012), "A novel bandwidth estimation algorithm for IEEE 802.11 TCP data transmissions", in IEEE wireless communications and networking conference workshops, 2012, pp. 377-382.

[74] Yuan, Z., "MBE: Model-based available bandwidth estimation for IEEE 802.11 data communications", in IEEE Transactions on Vehicular Technology, 2012, vol.6, no.5, pp.2158-2171. 